\title{
Improving the Readability of Pediatric Hospital Medicine Discharge Instructions
}

Ndidi I. Unaka, MD, MEd ${ }^{1 *}$, Angela Statile, MD, MEd¹, Karen Jerardi, MD, MEd ${ }^{1}$, Devesh Dahale, $\mathrm{MS}^{2}$, Joan Morris, RN, MBA, MHSA ${ }^{3}$, Brianna Liberio ${ }^{1}$, Ashley Jenkins, MD1, Blair Simpson, MD', Randi Mullaney, CNP ${ }^{1}$, Jodi Kelley, CNP ${ }^{1}$, Michelle Durling ${ }^{1}$, Jennifer Shafer ${ }^{1}$, Patrick W. Brady, MD, MSc ${ }^{1,2}$

${ }^{1}$ Division of Hospital Medicine, Cincinnati Children's Hospital Medical Center, Cincinnati, Ohio; 2James M. Anderson Center for Health Systems Excellence, Cincinnati Children's Hospital Medical Center, Cincinnati, Ohio; ${ }^{3}$ Center for Professional Excellence/Education, Cincinnati Children's Hospital Medical Center, Cincinnati, Ohio.

BACKGROUND: Readable discharge instructions may help caregivers understand and implement care plans following hospitalization. Many caregivers of hospitalized children, however, have limited literacy. We aimed to increase the percentage of discharge instructions written at 7 th grade level or lower for hospital medicine patients from $13 \%$ to $80 \%$ in 6 months.

METHODS: Quality improvement efforts targeted a 42-bed unit at the community satellite of our large, urban academic hospital. A multidisciplinary team of physicians, nurses, and parents focused on key drivers: family engagement in discharge process, standardization of discharge instructions, staff engagement in discharge preparedness, and audit and feedback of data. Improvement cycles included 1) education and implementation of a general discharge instruction template in the electronic health record (EHR); 2) visible reminders and tips for writing readable discharge instructions;
3) implementation of disease-specific discharge instruction templates in the EHR; and 4) individualized feedback to staff on readability and content of their written discharge instructions. Instructions were individually scored for readability using an online platform. An annotated control chart assessed the impact of interventions over time.

RESULTS: Through sequential interventions over 6 months, the percentage of discharge instructions written at 7 th grade or lower readability level increased from $13 \%$ to $98 \%$ and has been sustained for 4 months. The reliable use of the EHR templates was associated with our largest improvements.

CONCLUSION: Use of standardized discharge instruction templates and rapid feedback to staff improved the readability of instructions. Next steps include adaptation and spread to other patient populations. Journal of Hospital Medicine 2017;12:551-557. (C) 2017 Society of Hospital Medicine
The transition from hospital to home can be overwhelming for caregivers. ${ }^{1}$ Stress of hospitalization coupled with the expectation of families to execute postdischarge care plans make understandable discharge communication critical. Communication failures, inadequate education, absence of caregiver confidence, and lack of clarity regarding care plans may prohibit smooth transitions and lead to adverse postdischarge outcomes. ${ }^{2-4}$

Health literacy plays a pivotal role in caregivers' capacity to navigate the healthcare system, comprehend, and execute care plans. An estimated 90 million Americans have limited health literacy that may negatively impact the provision of safe and quality care ${ }^{5,6}$ and be a risk factor for poor outcomes, including increased emergency department (ED) utilization and readmission rates. ${ }^{7-9}$ Readability strongly influences the effectiveness of written materials. ${ }^{10}$ However,

\footnotetext{
*Address for correspondence and reprint requests: Ndidi I. Unaka, Division of Hospital Medicine, Cincinnati Children's Hospital Medical Center, 3333 Burnet Ave., ML 5018, Cincinnati, OH 45229; Telephone: 513-636-8354; Fax: 513636-7905; E-mail: ndidi.unaka@cchmc.org

Additional Supporting Information may be found in the online version of this article.

Received: December 7, 2016; Revised: February 1, 2017; Accepted: February 5, 2017
}

2017 Society of Hospital Medicine DOI 10.12788/jhm.2770 written medical information for patients and families are frequently between the $10^{\text {th }}$ and $12^{\text {th }}$ grade reading levels; more than $75 \%$ of all pediatric health information is written at or above $10^{\text {th }}$ grade reading level. ${ }^{11}$ Government agencies recommend between a $6^{\text {th }}$ and $8^{\text {th }}$ grade reading level, for written material; $5,12,13$ written discharge instructions have been identified as an important quality metric for hospital-to-home transitions. ${ }^{14-16}$

At our center, we found that discharge instructions were commonly written at high reading levels and often incomplete. ${ }^{17}$ Poor discharge instructions may contribute to increased readmission rates and unnecessary ED visits. ${ }^{9,18}$ Our global aim targeted improved health-literate written information, including understandability and completeness.

Our specific aim was to increase the percentage of discharge instructions written at or below the 7 th grade level for hospital medicine (HM) patients on a community hospital pediatric unit from $13 \%$ to $80 \%$ in 6 months.

\section{METHODS}

\section{Context}

The improvement work took place at a 42-bed inpatient pediatric unit at a community satellite of our large, urban, academic hospital. The unit is staffed by medical providers including attendings, fellows, nurse practitioners (NPs), and senior pediatric residents, and had more than $1000 \mathrm{HM}$ discharges 


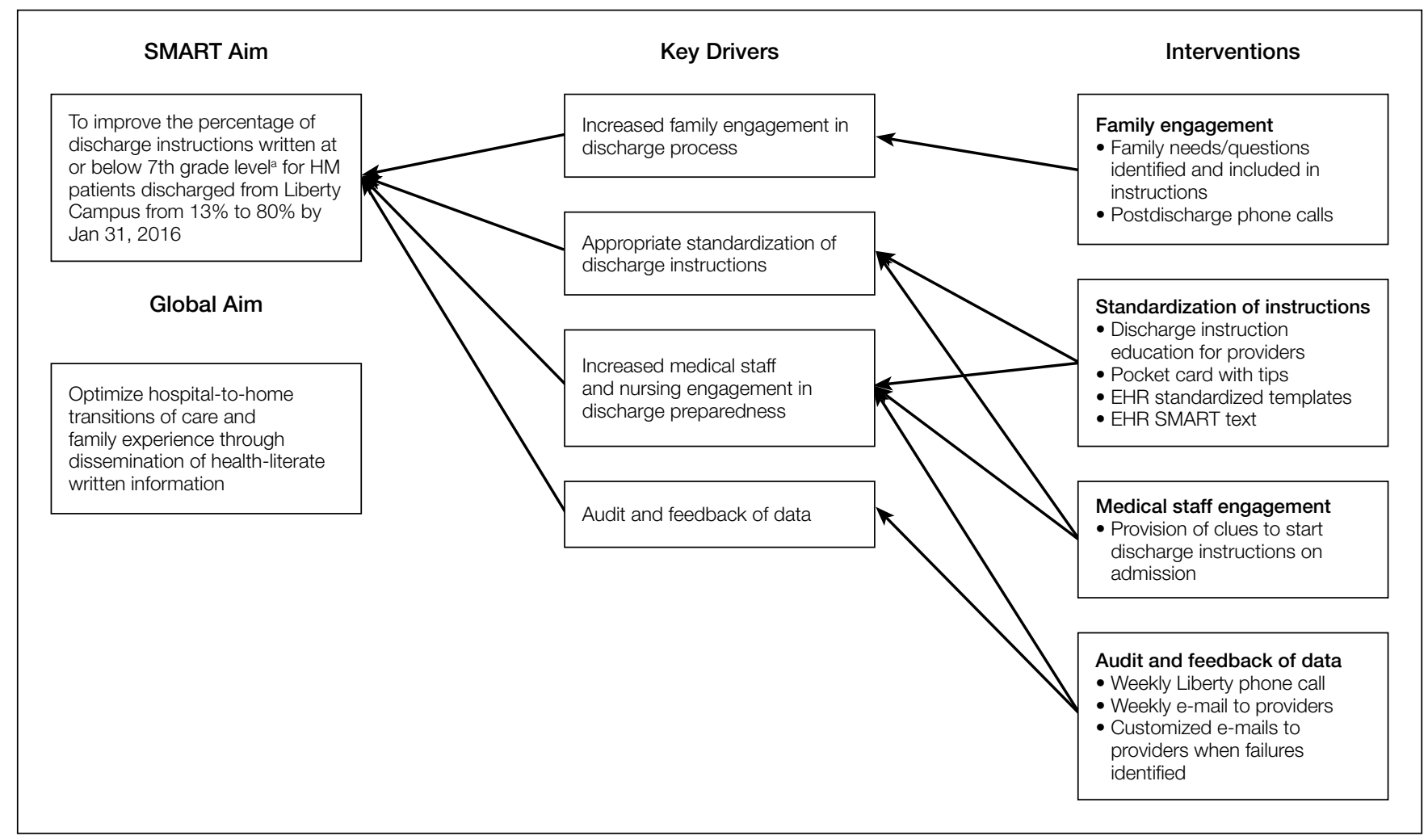

FIG 1. Key driver diagram.

aReadability measured by mean readability score calculated from the following formulas: Flesch Kincaid Grade Level, Simple Measure of Gobbledygook Index, Coleman-Liau Index, Gunning-Fog Index and Automated Readability Index.

Note: Abbreviations: EHR, electronic health record; HM, hospital medicine.

in fiscal year 2016. Children with common general pediatric diagnoses are admitted to this service; postsurgical patients are not admitted primarily to the HM service. In Cincinnati, the neighborhood-level high school drop-out rates are as high as $64 \% .{ }^{19}$ Discharge instructions are written by medical providers in the electronic health record (EHR). A printed copy is given to families and verbally reviewed by a bedside nurse prior to discharge. Quality improvement (QI) efforts focused on discharge instructions were ignited by a prior review of 200 discharge instructions that showed they were difficult to read (median reading level of $10^{\text {th }}$ grade), poorly understandable (36\% of instructions met the threshold of understandability as measured by the Patient Education Materials Assessment $\mathrm{Tool}^{20}$ ) and were missing key elements of information. ${ }^{17}$

\section{Improvement Team}

The improvement team consisted of 4 pediatric hospitalists, 2 NPs, 1 nurse educator with health literacy expertise, 1 pediatric resident, 1 fourth-year medical student, 1 QI consultant, and 2 parents who had first-hand experience on the HM service. The improvement team observed the discharge process, including roles of the provider, nurse and family, outlined a process map, and created a modified failure mode and effect analysis. ${ }^{21}$ Prior to our work, discharge instructions written by providers often occurred as a last step, and the content was created as free text or from nonstandardized templates. Key drivers that informed interventions were determined and revised over time (Figure 1). The study was reviewed by our institutional review board and deemed not human subjects research.

\section{Improvement Activities}

Key drivers were identified, and interventions were executed using Plan-Do Study-Act cycles. ${ }^{22}$ The key drivers thought to be critical for the success of the QI efforts were family engagement; standardization of discharge instructions; medical staff engagement; and audit and feedback of data. The corresponding interventions were as follows:

\section{Family Engagement}

Understanding the discharge information families desired. Prior to testing, 10 families admitted to the HM service were asked about the discharge experience. We asked families about information they wanted in written discharge instructions: 1$)$ reasons to call your primary doctor or return to the hospital; 2) when to see your primary doctor for a follow-up visit; 3) the phone number to reach your child's doctor; 4) more information about why your child was admitted; 5) information about new medications; and 6) what to do to help your child continue to recover at home.

Development of templates. We engaged families throughout the process of creating general and disease-specific dis- 
Abigail was in the hospital for anaphylaxis, which is a very bad allergic reaction. A child with anaphylaxis can have hives, swelling of lips or eyes, vomiting, and/or trouble breathing.

What you can do at home to make sure Abigail stays healthy:

- Keep what causes the allergic reaction away from Abigail

- If a food caused the allergic reaction:

o Tell everyone who takes care of Abigail not to give your child that food.

- Ask about what ingredients are in a food item if you are not sure.

o Read all food labels carefully.

- Do not give Abigail foods that are "manufactured on the same equipment" or "manufactured in a facility" that makes the food your child is allergic to.

o When she is eating away from home (for example, at a restaurant or a friend's house), tell everyone about her allergy.

- If Abigail is allergic to a medicine, do not give her the medicine.

- Make sure school and/or daycare know about her allergy and what to do in an emergency.

- Make sure an Epinephrine shot is with Abigail AT ALL TIMES.

Abigail needs to take these medicines in order to get better:

- If she has hives (an itchy red rash) give Benadry| ${ }^{\star \star * 1}$ every 6 hours as needed.

We would like Abigail to see Dr. Smith in ${ }^{\star \star \star}$ days. Please call Abigail's doctor at XXX-XXX-XXXX to make an appointment

If Abigail has any of these signs, call the doctor for advice. Abigail may need to see the doctor if: - Medicine is not helping the itchy rash (hives).

Give Abigail the Epinephrine shot in the thigh AND go to the emergency room/call 911 if she has any of these signs:

- Any swelling in the face (eyes, lips, tongue, throat).

- Trouble breathing (belly, rib, or neck muscles are going up and down while breathing).

- Wheezing.

- Feel faint or lightheaded.

- Vomiting.

- Looks pale or you see a blue color around her mouth.
Discharge diagnosis

with description

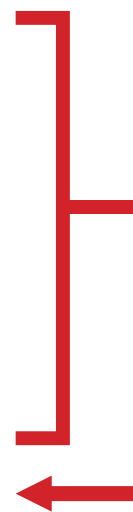

Postdischarge care tips and new medications

PCP follow-up instructions with content info

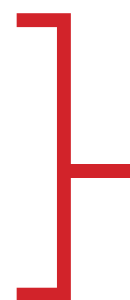

Red flag and escalation plan

FIG. 2. Disease-specific discharge instruction template.

charge templates. After a specific template was created and reviewed by the parents on our team, it was sent to members of the institutional Patient Education Committee, which includes parents and local health literacy experts, to review and critique. Feedback from the reviewers was incorporated into the templates prior to use in the EHR.

Postdischarge phone calls. A convenience sample of families discharged from the satellite campus was called 24 to 48 hours after discharge over a 2-week period in January, 2016. A member of our improvement team solicited feedback from families about the quality of the discharge instructions. Families were asked if discharge instructions were reviewed with them prior to going home, if they were given a copy of the instructions, how they would rate the ability to read and use the information, and if there were additional pieces of information that would have improved the instructions.

\section{Standardization of Instructions}

Education. A presentation was created and shared with medical providers; it was re-disseminated monthly to new residents rotating onto the service and to the attendings, fellows, and NPs scheduled for shifts during the month. This education continued for the duration of the study. The presentation included the definition of health literacy, scope of the problem, examples of poorly written discharge instructions, and tips on how to write readable and understandable instructions. Laminated cards that included tips on how to write instructions were also placed on work stations.

Creation of discharge instruction templates in the EHR.A general discharge instruction template that was initially created and tested in the EHR (Figure 2) included text written below the 7th grade and employed 14 point font, bolded words for emphasis, and lists with bullet points. Asterisks were used to indicate where providers needed to include patient-specific information. The sections included in the general template were informed by feedback from providers and parents prior to testing, parents on the improvement team, and parents of patients admitted to our satellite campus. The sections reflect components critical to successful postdischarge care: discharge diagnosis and its brief description, postdischarge care information, new medications, signs and symptoms that would warrant escalation of care to the patient's primary care provider or the ED, and follow-up instructions and contact information for the patent's primary care doctor.

While the general template was an important first step, the content relied heavily on free text by providers, which could still lead to instructions written at a high reading level. Thus, disease-specific discharge instruction templates were created with prepopulated information that was written at a reading level at or below $7^{\text {th }}$ grade level (Figure 2). The diseases were prioritized based on the most common diagnoses on our HM service. Each template included information under each of the subheadings noted in the general template. Twelve disease-specific templates were tested and ultimately embedded in 


\section{TABLE. Patient Characteristics}

\begin{tabular}{ll}
\hline Characteristic & $\mathrm{N}(\%)$ \\
\hline Female & $997(50)$ \\
\hline Mean age in years (SD) & $6.7(6.1)$ \\
\hline Mean length of stay in hours (SD) & $34.5(27.3)$ \\
\hline Publicly insured & $942(47)$ \\
\hline Most common discharge diagnoses & \\
Acute bronchiolitis & $241(12)$ \\
Asthma exacerbation & $192(10)$ \\
Pneumonia (viral + bacterial) & $157(8)$ \\
Viral upper respiratory infection & $87(4)$ \\
Dehydration & $86(4)$ \\
Constipation & $82(4)$ \\
Croup & $68(3)$ \\
Gastroenteritis & $51(2)$ \\
Urinary tract infection & $48(2)$ \\
\hline
\end{tabular}

NOTE: Abbreviation: SD, standard deviation.

the EHR; the general template remained for use when the discharge diagnosis was not covered by a disease-specific template.

\section{Medical Staff Engagement}

Previously described tests of change also aimed to enhance staff engagement. These included frequent e-mails, discussion of the QI efforts at specific team meetings, and the creation of visual cues posted at computer work stations, which prompted staff to begin to work on discharge instructions soon after admission.

\section{Audit and Feedback of Data}

Weekly phone calls. One team updated clinicians through a regularly scheduled bi-weekly phone conference. The phone conference was established prior to our work and was designed to relay pertinent information to attendings and NPs who work at the satellite hospital. During the phone conferences, clinicians were notified of current performance on discharge instruction readability and specific tests of change for the week. Additionally, providers gave feedback about the improvement efforts. These updates continued for the first 6 months of the project until sustained improvements were observed.

E-mails. Weekly e-mails were sent to all providers scheduled for clinical time at the satellite campus. The e-mail contained information on current tests of change, a list of discharge instruction templates that were available in the EHR, and the annotated run chart illustrating readability levels over time.

Additionally, individual e-mails were sent to each provider after review of the written discharge instructions for the week. Providers were given information on the number of discharge instructions they personally composed, the percentage of those instructions that were written at or below $7^{\text {th }}$ grade level, and specific feedback on how their written instructions could be improved. We also encouraged feedback from each provider to better identify barriers to achieving our goal.

\section{Study of the Interventions}

Baseline data included a review of all instructions for patients discharged from the satellite campus from the end of April 2015 through mid-September 2015. The time period for testing of interventions during the fall and winter months allowed for rapid cycle learning due to higher patient census and predictability of admissions for specific diagnosis (ie, asthma and bronchiolitis). An automated report was generated from the EHR weekly with specific demographics and identifiers for patient discharged over the past 7 days, including patient age, gender, length of stay, discharge diagnosis, and insurance classification. Data was collected during the intervention period via structured review of the discharge instructions in the EHR by the principal investigator or a trained research coordinator. Discharge instructions for medically cleared mental health patients admitted to hospital medicine while awaiting psychiatric bed availability and patients and parents who were non-English speaking were excluded from review. All other instructions for patients discharged from the HM service at our Liberty Campus were included for review.

\section{Measures}

Readability, our primary measure of interest, was calculated using the mean score from the following formulas: Flesch Kincaid Grade Level, ${ }^{23}$ Simple Measure of Gobbledygook Index, ${ }^{24}$ Coleman-Liau Index, ${ }^{25}$ Gunning-Fog Index, ${ }^{26}$ and Automated Readability Index ${ }^{27}$ by means of an online platform (https://readability-score.com). ${ }^{28}$ This platform was chosen because it incorporated a variety of formulas, was user-friendly, and required minimal data cleaning. Each of the readability formulas have been used to assesses readability of health information given to patients and families. ${ }^{29,30}$ The threshold of 7 th grade is in alignment with our institutional policy for educational materials and with recommendations from several government agencies. ${ }^{5,12}$

\section{Analysis}

A statistical process control p-chart was used to analyze our primary measure of readability, dichotomized as percent discharge instructions written at or below 7th grade level. Run charts were used to follow mean reading level of discharge instructions and our process measure of percent of discharge instruction written with a general or disease-specific standardized template. Run chart and control chart rules for identifying special cause were used for midline shifts. ${ }^{31}$

\section{RESULTS}

The Table includes the demographic and clinical information of patients included in our analyses. Through sequential interventions, the percentage of discharge instructions written at or below 7th grade readability level increased from a mean of $13 \%$ to more than $80 \%$ in 3 months (Figure 


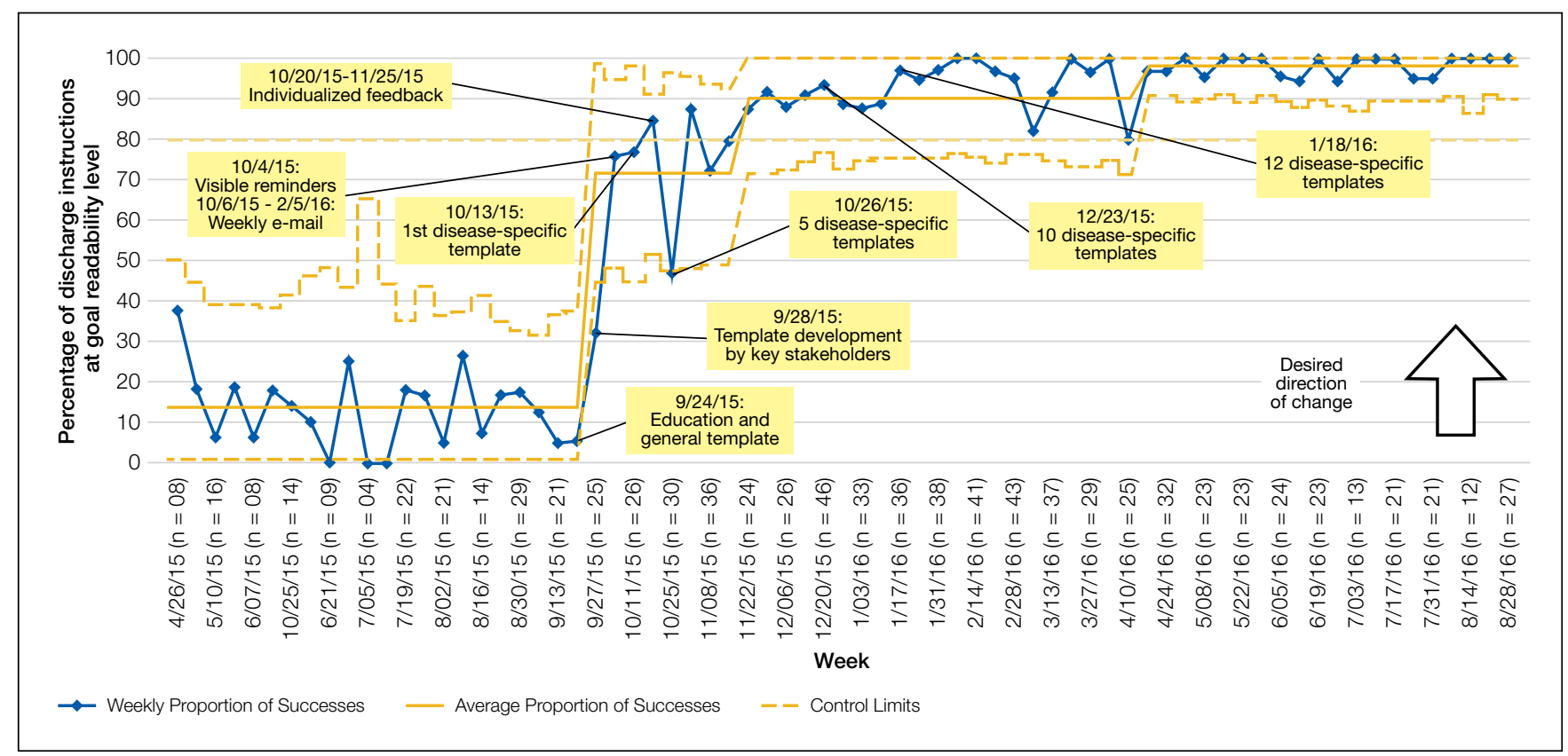

FIG 3. Percentage of discharge instructions written at or below $7^{\text {th }}$ grade readability level.

3). Furthermore, the mean was sustained above $90 \%$ for 10 months and at $98 \%$ for the last 4 months. The use of 1 of the 13 EHR templates increased from $0 \%$ to $96 \%$ and was associated with the largest impact on the overall improvements (Supplemental Figure 1). Additionally, the average reading level of the discharge instructions decreased from 10th grade to 6th grade level (Supplemental Figure 2).

Qualitative comments from providers about the discharge instructions included:

"Are these [discharge instructions] available at base?? Great resource for interns."

"These [discharge] instructions make the [discharge] process so easy!!! Love these..."

"Also feel like they have helped my discharge teaching in the room!"

Qualitative comments from families postdischarge included:

"I thought the instructions were very clear and easy to read. I especially thought that highlighting the important areas really helped."

"I think this form looks great, and I really like the idea of having your child's name on it."

\section{DISCUSSION}

Through sequential Plan-Do Study-Act cycles, we increased the percentage of discharge instructions written at or below $7^{\text {th }}$ grade reading level from $13 \%$ to $98 \%$. Our most impactful intervention was the creation and dissemination of standardized disease-specific discharge instruction templates. Our findings complement evidence in the adult and pediatric literature that the use of standardized, disease-specific discharge instruction templates may improve readability of instructions. ${ }^{32,33}$ And, while quality improvement efforts have been employed to improve the discharge process for patients, ${ }^{34-36}$ this is the first study in the inpatient setting that, to our knowledge, specifically addresses discharge instructions using quality improvement methods.

Our work targeted the critical intersection between individual health literacy, an individual's capacity to acquire, interpret, and use health information, and the necessary changes needed within our healthcare system to ensure that appropriately written instructions are given to patients and families. ${ }^{17,37}$ Our efforts focused on improving discharge instructions answer the call to consider health literacy a modifiable clinical risk factor. ${ }^{37}$ Furthermore, we address the 6 aims for quality healthcare delivery: 1) safe, timely, efficient and equitable delivery of care through the creation and dissemination of standardized instructions that are written at the appropriate reading level for families to ease hospital-to-home transitions and streamline the workflow of medical providers; 2) effective education of medical providers on health literacy concepts; and 3) family-centeredness through the involvement of families in our QI efforts. While previous QI efforts to improve hospital-to-home transitions have focused on medication reconciliation, communication with primary care physicians, follow-up appointments, and timely discharges of patients, none have specifically focused on the quality of discharge instructions. ${ }^{34-36}$

Most physicians do not receive education about how to write information that is readable and understandable; more than half of providers desired more education in this area. ${ }^{38}$ Furthermore, pediatric providers may overestimate parental health literacy levels, ${ }^{39}$ which may contribute to variability in the readability of written health materials. While education alone can contribute to a provider's ability to create readable instructions, we note the improvement after the 
introduction of disease templates to demonstrate the importance of workflow-integrated higher reliability interventions to sustain improvements.

Our baseline poor readability rates were due to limited knowledge by frontline providers composing the instructions and a system in which an important element for successful hospital-to-home transitions was not tackled until patients were ready for discharge. Streamlining of the discharge process, including the creation of discharge instructions, may lead to improved efficiency, fewer discrepancies, more effective communication, and an enhanced family experience. Moreover, the success of our improvement work was due to key stakeholders, including parents, being a part of the team and the notable buy-in from providers.

Our work was not without limitations. We excluded non-English speaking families from the study. We were unable to measure reading level of our population directly and instead based our goals on national estimates. Our primary measure was readability, which is only 1 piece contributing to quality discharge instructions. Understandability and actionability are also important considerations; ${ }^{17,20,29,40}$ however, improvements in these areas were limited by our design options within the EHR. Our efforts focused on children with common general pediatric diagnoses, and it is unclear how our interventions would generalize to medically complex patients with more volume of information to communicate at discharge and with uncommon diagnoses that are less readily incorporated into standardized templates. Relatedly, our work occurred at the satellite campus of our tertiary care center and may not represent generalizable material or methods to implement templates at our main campus location or at other hospitals. To begin to better understand this, we have spread to HM patients at our main campus, including medically complex patients with technology dependence and/or neurological impairments. Standardized, disease-specific templates most relevant to this population as well as several patient specific templates, for those with frequent readmissions due to medical complexity, have been created and are actively being tested.

\section{CONCLUSION}

In conclusion, in using interventions targeted at standardization of discharge instructions and timely feedback to staff, we saw rapid, dramatic, and sustained improvement in the readability of discharge instructions. Next steps include adaptation and spread to other patient populations and care teams, collaborations with other centers, and assessing the impact of effectively written discharge instructions on patient outcomes, such as adverse drug events, readmission rates, and family experience.

Disclosure: No external funding was secured for this study. Dr. Brady is supported by a Patient-Centered Outcomes Research Mentored Clinical Investigator Award from the Agency for Healthcare Research and Quality, Award Number K08HS023827. The content is solely the responsibility of the authors and does not necessarily represent the official views of the funding organizations. The funding organization had no role in the design, preparation, review, or approval of this paper; nor the decision to submit the manuscript for publication. The authors have no financial relationships relevant to this article to disclose.

\section{References}

1. Solan LG, Beck AF, Brunswick SA, et al. The family perspective on hospital to home transitions: a qualitative study. Pediatrics. 2015;136:e1539-e1549.

2. Engel KG, Buckley BA, Forth VE, et al. Patient understanding of emergency department discharge instructions: where are knowledge deficits greatest? Acad Emerg Med. 2012;19:E1035-E1044.

3. Ashbrook L, Mourad M, Sehgal N. Communicating discharge instructions to patients: a survey of nurse, intern, and hospitalist practices. J Hosp Med. 2013;8: $36-41$

4. Kripalani S, Jacobson TA, Mugalla IC, Cawthon CR, Niesner KJ, Vaccarino V. Health literacy and the quality of physician-patient communication during hospitalization. J Hosp Med. 2010;5:269-275.

5. Institute of Medicine Committee on Health Literacy. Kindig D, Alfonso D, Chudler E, et al, eds. Health Literacy: A Prescription to End Confusion. Washington, DC: National Academies Press; 2004.

6. Yin HS, Johnson M, Mendelsohn AL, Abrams MA, Sanders LM, Dreyer BP. The health literacy of parents in the United States: a nationally representative study. Pediatrics. 2009;124(suppl 3):S289-S298.

7. Rak EC, Hooper SR, Belsante MJ, et al. Caregiver word reading literacy and health outcomes among children treated in a pediatric nephrology practice. Clin Kid J. 2016;9:510-515.

8. Morrison AK, Schapira MM, Gorelick MH, Hoffmann RG, Brousseau DC. Low caregiver health literacy is associated with higher pediatric emergency department use and nonurgent visits. Acad Pediatr. 2014;14:309-314.

9. Howard-Anderson J, Busuttil A, Lonowski S, Vangala S, Afsar-Manesh N. From discharge to readmission: Understanding the process from the patient perspective. J Hosp Med. 2016;11:407-412.

10. Doak CC, Doak LG, Root JH. Teaching Patients with Low Literacy Skills. 2nd ed. Philadelphia PA: J.B. Lippincott; 1996.

11. Berkman ND, Sheridan SL, Donahue KE, et al. Health literacy interventions and outcomes: an updated systematic review. Evid Rep/Technol Assess. 2011;199:1-941.

12. Prevention CfDCa. Health Literacy for Public Health Professionals. In: Prevention CfDCa, ed. Atlanta, GA2009.

13. "What Did the Doctor Say?" Improving Health Literacy to Protect Patient Safety. Oakbrook Terrace, IL: The Joint Commission, 2007.

14. Desai AD, Burkhart Q, Parast L, et al. Development and pilot testing of caregiver-reported pediatric quality measures for transitions between sites of care. Acad Pediatr. 2016;16:760-769.

15. Leyenaar JK, Desai AD, Burkhart Q, et al. Quality measures to assess care transitions for hospitalized children. Pediatrics. 2016;138(2).

16. Akinsola B, Cheng J, Zmitrovich A, Khan N, Jain S. Improving discharge instructions in a pediatric emergency department: impact of a quality initiative. Pediatr Emerg Care. 2017;33:10-13.

17. Unaka NI, Statile AM, Haney J, Beck AF, Brady PW, Jerardi K. Assessment of the readability, understandability and completeness of pediatric hospital medicine discharge instructions J Hosp Med. In press.

18. Stella SA, Allyn R, Keniston A, et al. Postdischarge problems identified by telephone calls to an advice line. J Hosp Med. 2014;9:695-699.

19. Maloney M, Auffrey C. The social areas of Cincinnati.

20. The Patient Education Materials Assessment Tool (PEMAT) and User's Guide: An Instrument To Assess the Understandability and Actionability of Print and Audiovisual Patient Education Materials. Available at: http://www.ahrq.gov/ professionals/prevention-chronic-care/improve/self-mgmt/pemat/index.html. Accessed November 27, 2013.

21. Cohen MR, Senders J, Davis NM. Failure mode and effects analysis: a novel approach to avoiding dangerous medication errors and accidents. Hosp Pharm. 1994;29:319-30.

22. Langley GJ, Moen R, Nolan KM, Nolan TW, Norman CL, Provost LP. The Improvement Guide: A Practical Approach to Enhancing Organizational Performance. San Franciso, CA: John Wiley \& Sons; 2009.

23. Flesch R. A new readability yardstick. J Appl Psychol. 1948;32:221-233.

24. McLaughlin GH. SMOG grading-a new readability formula. J Reading. 1969;12:639-646.

25. Coleman M, Liau TL. A computer readability formula designed for machine scoring. J Appl Psych. 1975;60:283.

26. Gunning R. \{The Technique of Clear Writing\}. 1952.

27. Smith EA, Senter R. Automated readability index. AMRL-TR Aerospace Medical Research Laboratories (6570th) 1967:1

28. How readable is your writing. 2011. https://readability-score.com. Accessed September 23, 2016. 
29. Yin HS, Gupta RS, Tomopoulos S, et al. Readability, suitability, and characteristics of asthma action plans: examination of factors that may impair understanding. Pediatrics. 2013;131:e116-E126.

30. Brigo F, Otte WM, Igwe SC, Tezzon F, Nardone R. Clearly written, easily comprehended? The readability of websites providing information on epilepsy. Epilepsy Behav. 2015;44:35-39.

31. Benneyan JC. Use and interpretation of statistical quality control charts. Int J Qual Health Care. 1998;10:69-73.

32. Mueller SK, Giannelli K, Boxer R, Schnipper JL. Readability of patient discharge instructions with and without the use of electronically available disease-specific templates. J Am Med Inform Assoc. 2015;22:857-863.

33. Lauster CD, Gibson JM, DiNella JV, DiNardo M, Korytkowski MT, Donihi AC Implementation of standardized instructions for insulin at hospital discharge. J Hosp Med. 2009;4:E41-E42.

34. Tuso P, Huynh DN, Garofalo L, et al. The readmission reduction program of Kaiser Permanente Southern California-knowledge transfer and performance improvement. Perm J. 2013;17:58-63.
35. White CM, Statile AM, White DL, et al. Using quality improvement to optimise paediatric discharge efficiency. BMJ Qual Saf. 2014;23:428-436.

36. Mussman GM, Vossmeyer MT, Brady PW, Warrick DM, Simmons JM, White CM. Improving the reliability of verbal communication between primary care physicians and pediatric hospitalists at hospital discharge. J Hosp Med. 2015;10:574580 .

37. Rothman RL, Yin HS, Mulvaney S, Co JP, Homer C, Lannon C. Health literacy and quality: focus on chronic illness care and patient safety. Pediatrics 2009;124(suppl 3):S315-S326.

38. Turner T, Cull WL, Bayldon B, et al. Pediatricians and health literacy: descriptive results from a national survey. Pediatrics. 2009;124(suppl 3):S299-S305.

39. Harrington KF, Haven KM, Bailey WC, Gerald LB. Provider perceptions of parent health literacy and effect on asthma treatment: recommendations and instructions. Pediatr Allergy immunol Pulmonol. 2013;26:69-75.

40. Yin HS, Parker RM, Wolf MS, et al. Health literacy assessment of labeling of pediatric nonprescription medications: examination of characteristics that may impair parent understanding. Acad Pediatr. 2012;12:288-296. 